\title{
บUisuersily
}

\section{GPSDTN: Predictive Velocity-Enabled Delay-Tolerant Networks for Arctic Research and Sustainability}

Beck, R., Hinkel, K., Eisner, W., Liu, L., Fall, K., Burleigh, S., Torgerson, L., Hooke, A., Durst, R., Pleva, D.,

Ivancic, W., Paulsen, P., Lambert, C., Groves, S., LeBrun, J., Norda, J., Li, J., Garuba, M., Bulger, R., ... Maffei, A. (2007). GPSDTN: Predictive Velocity-Enabled Delay-Tolerant Networks for Arctic Research and

Sustainability. In Unknown Host Publication IEEE. https://doi.org/10.1109/ICIMP.2007.20

Link to publication record in Ulster University Research Portal

Published in:

Unknown Host Publication

Publication Status:

Published (in print/issue): 01/07/2007

DOI:

10.1109/ICIMP.2007.20

Document Version

Publisher's PDF, also known as Version of record

\section{General rights}

Copyright for the publications made accessible via Ulster University's Research Portal is retained by the author(s) and / or other copyright owners and it is a condition of accessing these publications that users recognise and abide by the legal requirements associated with these rights.

\section{Take down policy}

The Research Portal is Ulster University's institutional repository that provides access to Ulster's research outputs. Every effort has been made to ensure that content in the Research Portal does not infringe any person's rights, or applicable UK laws. If you discover content in the Research Portal that you believe breaches copyright or violates any law, please contact pure-support@ulster.ac.uk. 


\section{GPSDTN: Predictive Velocity-Enabled Delay-Tolerant Networks for Arctic Research and Sustainability}

\author{
R. Beck, K. Hinkel, W. Eisner, \\ L. Liu, Univ. Cincinnati, USA \\ richard.beck@uc.edu
}

Kevin Fall
Intel Research
Berkeley, USA

S. Burleigh, L. Torgerson,

A. Hooke, NASA JPL

Pasadena, USA
Robert Durst
Mitre Corporation
McLean, USA

D. Pleva, W. Ivancic, P. Paulsen

NASA Glenn Research Center

Cleveland, USA
Charles Lambert, Steven Groves
SkySentry LLC
Colorado Springs, USA

Jason LeBrun

University of California

Davis, USA

\author{
Jacob Norda \\ Trimble Navigation Limited \\ Sunnyvale, USA
}

Jiang Li, Moses Garuba
Howard University
Washington, USA

Robert Bulger, Glenn Sheehan

Barrow Arctic Science

Barrow, USA

Avri Doria, Maria Uden

Lulea University of Technology

Lulea, Sweden

$$
\begin{aligned}
& \text { Ward Bathrick } \\
& \text { Raytheon } \\
& \text { Fullerton, USA }
\end{aligned}
$$
Christopher Small
BBN Technologies
Boston, USA

Marc Seibert

NASA Kennedy Space Center

Florida, USA

\author{
Ngoc Hoang \\ NAL Research Corporation \\ Manassas, USA
}

Richard Machida, Steve Smith

University of Alaska at Fairbanks

Fairbanks, USA.

Ben Ellis

Institute of the North

Anchorage, USA

\section{James Ferl \\ Chartracer \\ Milton, USA}

G. Parr, C. Peoples, B. Scotney,

A. Moore, University of Ulster

Coleraine, UK

\section{Lawrence Freudinger \\ NASA Dryden Research Center \\ California, USA}

Andrew Maffei

Woods Hole Oceanographic Inst.
Abstract

A Delay-Tolerant Network (DTN) is a necessity for communication nodes that may need to wait for long periods to form networks. The IETF Delay Tolerant Network Research Group is developing protocols to enable such networks for a broad variety of Earth and interplanetary applications. The Arctic would benefit from a predictive velocity-enabled version of DTN that would facilitate communications between sparse, ephemeral, often mobile and extremely power-limited nodes. We propose to augment DTN with power-aware, buffer-aware location- and time-based predictive routing for ad-hoc meshes to create networks that are inherently location and time (velocity) aware at the network level to support climate research, emergency services and rural education in the Arctic. On Earth, the primary source of location and universal time information for networks is the Global Positioning System (GPS). We refer to this Arctic velocity-enabled Delay-Tolerant Network protocol as "GPSDTN" accordingly. This paper describes our requirements analysis and general implementation strategy for GPSDTN to support Arctic research and sustainability efforts.

Keywords-(Delay-Tolerant Network, DTN, MANET, GPS, Predictive Routing, Velocity-Enabled Routing, Arctic)

\section{INTRODUCTION}

We propose to begin to unite the circumpolar community with a velocity-enabled version of the Delay Tolerant Network (DTN) protocol [1] that we refer to as GPSDTN [2]. GPSDTN is designed to leverage watercraft, aircraft and snow machine traffic to improve connectivity to and within the Arctic. Our 
project will begin with the Sami people of northern Sweden and Alaskan Natives on the North Slope of Alaska. They represent some of the most isolated and underserved groups of Arctic inhabitants [3,4]. The Sami and Alaskan Natives are representative user communities for the telecommunications needs of the Arctic in general. Both communities lack significant road and cable access to the rest of the world. Both areas are also characterized by severe weather and oceanic conditions that frequently prohibit air or sea travel, resulting in complete isolation during times when access is most crucial such as medical emergencies [4].

Declining government spending and decreases in fossil fuel production are resulting in decreased economic opportunities for a number of Arctic people, while others are affected by increased competition over land and water with limited possibilities to keep up the level of income from the present ways of organizing traditional sources of income and subsistence [3]. Access to the Internet carries potential for increasing the incomes through making genuine business cases, so that a traditional life style would no longer be a hindrance for staying in contact with major markets [3]. Increasing scientific research and support opportunities in these areas is another method and there is a pressing arctic research reason to do so. Traditional Knowledge is now playing an important role in mainstream, peer-reviewed, quantitative Arctic climate research [5]. No one knows the Arctic better than its indigenous peoples. Unfortunately, both educational and scientific research support opportunities are severely limited by poor wireless telecommunications and a rapidly decaying scientific research support infrastructure in these areas, inhibiting Arctic research accordingly $[4,6]$.

GPSDTN addresses the first problem; isolation and the need to develop a robust and redundant wireless delay tolerant network to support circumpolar research, education; telemedicine; municipal, federal, and public access to the Internet via hybrid terrestrial wireless and wired, ground-, space- and atmospherically-based wireless network technologies. Meetings with representatives from six arctic nations have resulted in a set of requirements for a wireless arctic network to unite the circumpolar community [4]. GPSDTN's initial focus will be monitoring climate change with a combination of traditional knowledge and low-cost sensor networks [5,7] as a testbed for circumpolar and interplanetary networks[7,9]. GPSDTN will begin with two nations, Sweden and the United States during the technology development phase. Once GPSDTN standards have been formalized, we will work to include all Arctic nations via the Northern Forum and the Arctic Council.

\section{REQUIREMENTS FOR ARCTIC RESEARCH AND SUSTAINABILITY DELAY- TOLERANT NETWORKS}

\section{A. General Requirements for Arctic DTNs}

We have established the following general requirements for wireless networks for Arctic research and sustainability efforts (Beck et al. 2005a):

- Directionality - 2-way, symmetric bandwidth.

Supported by the National Science Foundation and Northern Forum
- Reliability - fully redundant over two separate technologies

- Interoperability - TCP/IP compatible protocols adapted for mixed-latency delay-tolerant networks and architectures for seamless hand-off between networks. This capability is especially important for satellite mesh networks such as Iridium ${ }^{\mathrm{TM}}$ where the number of satellite hops and downlink locations may vary and users are likely to be mobile.

- Security - messages must be relayed without eavesdropping.

- Logistics - All ground systems must be cargo aircraft portable.

- Temperature Range -+38 to $-51{ }^{\circ} \mathrm{C}$.

- Bandwidth - Need for interoperable, integrated high $(>128 \mathrm{kbs})$ and low $(<128 \mathrm{kbs})$ bandwidth networks.

- Signaling - A signaling facet within the protocol to tell reverse nodes of network congestion or loss of upstream capacity or connections so that those nodes can enter a limited store mode until the network is reestablished or paths are redirected.

\section{B. Specific Requirements for Circumpolar Arctic Research and Sustainability Networks}

The GPSDTN subgroup has identified the following specific requirements for Arctic DTNs.

- Industrial strength reliability - Iridium ${ }^{\mathrm{TM}}$ Mesh Networks for the narrowband velocity-enabled routing channel and carrier-grade Internet access for coordinating "supernodes" that connect GPSDTNs to the Internet located at Lulea Technical University in Sweden and at the Barrow Global Climate Change Research Facility (BGCCRF) in Barrow, Alaska, USA.

- Bandwidth - single channel Iridium ${ }^{\mathrm{TM}}$ routing channels for location-based routing and broadband local ad hoc mesh networks on tundra and sea.

- Development of rugged, portable, insulated, rechargeable, single-button (on, off, reset) GPSDTN nodes for Arctic research and sustainability efforts.

- Omni-directional antennae and 12/120/220 volt power for ease of use.

- $\quad$ Long-life power sources (365 days).

- Beacon Capability - Development of "wake-up" beacon capability for very low-power nodes in the Arctic.

- Emergency mode - an emergency operation mode for when the power levels are extremely low. The unit enters a low consumption mode where it only wakes-up for short periods and transmits a beacon, then the last known good location and other logical data and then powers down until the next cycle to rest the batteries. This would be 
useful for locating lost sensor or transit nodes that may drift or be moved by various means.

- Field testing in the Arctic with real applications such as sensor nets, FTP, web caching, and email.

\section{CURRENT ARCTIC COMMUNICATIONS - GEO SATELLITES}

GPSDTN is necessary because remote polar regions currently rely upon expensive Geosynchronous or Geostationary Earth Orbit (GEO) satellite relays for most of their communications in remote areas. GEO satellites that orbit the earth $36,000 \mathrm{~km}$ above the equator are low on the horizon in the Arctic, forcing a very long propagation path at a low elevation angle through the atmosphere that is often unstable. This instability in the propagation medium causes a timevarying index of refraction, which slightly refracts the propagating electromagnetic waves. The net result is known as scintillation, which is a relatively rapid increase and decrease of the received signal strength. Scintillations can impact communication link [10]. If the signals become too strong, the amount of inter-modulation can increase in a multi-carrier environment, such as a satellite transponder. As the signals attenuate, the reduced signal-to-noise ratio can impact the quality of data transmission and create an unacceptable bit error rate (BER). Scintillations increase as the elevation angle of the link decreases and as the frequency of the link increases, limiting the footprint of individual satellites.

In addition to being error-prone, polar communications via GEO satellites are also characterized by significant delays. The distances from users in polar regions to the GEO satellites are greater than $40,000 \mathrm{~km}$ resulting in delays of approximately one quarter of a second of round-trip travel time (RTT) [11]. Long cables, routers, switches and signal processing can increase this delay. These delays are significant because standard TCP/IP [12,13] does not perform well over highlatency (long delay) mediums due to its additive increase (AI) and multiplicative decrease (MD) algorithm. TCP probes available bandwidth linearly (and therefore slowly), while it decreases the sending rate exponentially using MD at congestion. Given long delays, such operations result in very high throughput, but low goodput on average. If the delay is too long, the connection will time-out and the applications that depend on the link may fail [11]. We need higher bandwidth delay-tolerant networks for external and local connectivity to support Arctic research and sustainability accordingly.

\section{Future ARCTIC COMMUNICATIONS - WiFI AND MOBLE WIMAX WITH LEO SATELLITE ROUTING CHANNELS FOR GPSDTN}

Low-Earth Orbit (LEO) satellites have orbits 485 to 1,414 $\mathrm{km}$ above the earth's surface. The signal propagation path involved when using LEOs is only a few percent of the geostationary path length. This allows the use of small, omnidirectional antennae and low power transmitters. Two LEO options exist for polar regions, Globalstar ${ }^{\mathrm{TM}}$ and Iridium $^{\mathrm{TM}}$. Globalstar $^{\mathrm{TM}}$ uses 48 satellites with orbits inclined at 52 degrees. This means that Globalstar ${ }^{\mathrm{TM}}$ coverage is concentrated at low- to mid-latitudes. Each Globalstar ${ }^{\mathrm{TM}}$ satellite operates as a bent-pipe repeater between the user and a ground station. Iridium ${ }^{\mathrm{TM}}$ uses 66 satellites with orbits inclined at 86.4 degrees. This means that Iridium $^{\mathrm{TM}}$ coverage is truly global and is especially good near the poles. Iridium ${ }^{\mathrm{TM}}$ satellites are linked to each other in a mesh network linked to ground stations in Tempe, Arizona and Fairbanks, Alaska. Iridium $^{\mathrm{TM}}$ includes a data service that provides up to $10 \mathrm{kbs}$ per channel via a data kit and a single Iridium ${ }^{\mathrm{TM}}$ phone. GPSDTN will incorporate the next generation of Iridium $^{\mathrm{TM}}$ single channel data terminals (that do not require a phone) and bonded higher bandwidth data terminals for low-volume web surfing, email, FTP etc. into an integrated sensor network for climate change research. Broad-band Iridium ${ }^{\mathrm{TM}}$ data service is being developed and expected to become commercially available by the $3^{\text {rd }}$ quarter of 2007. A new Iridium ${ }^{\text {TM }}$ data terminal slightly bigger in form-factor than the NAL Research's A3LA-DGS combined with a phase-array antenna can support data rates ranging from $64 \mathrm{kbits} / \mathrm{sec}$ to $128 \mathrm{kbits} / \mathrm{sec}$ (true bandwidth, noncompressed).

The standard TCP/IP stack can be adjusted at both ends of a link to significantly improve transmission efficiency over Arctic GEO or multi-hop LEO satellite links by a factor of at least four for large file transfers if the expected delay is known ahead of time $[4,11]$. If an 'adjusted' TCP is used in places other than the particular link it would compete unfairly with other TCP's. Proprietary alternatives to TCP/IP such as XTP have been developed for high-latency satellite links. With a dynamic mesh of LEO satellites that move relative to each other and relative to ground stations, the delay will vary and must be adapted for by the protocol itself. NASA Glenn's Satellite Communications group has tested some of these alternative protocols including XTP from Packeteer. These proprietary protocols usually require significant proprietary hardware at each end of the link to interface with standard TCP/IP in the rest of the network.

Non-proprietary CCSDS Space Communications Protocol Standards (SCPS) Gateways are freely available (both opensource and commercial GS Gateways are available.) A gateway approach has the advantage that all of the translation to/from the performance-enhancing space link protocols is transparent to all users outside the link and no local modification of enduser equipment is required. Fortunately, given that scaleable satellite mesh networks for remote areas in the Arctic will require inexpensive ways to convert standard TCP/IP traffic into mixed-latency, delay-tolerant network (DTN), TCP/IP compatible protocols $[1,14,15,16]$, the open-source CCSDS SCPS-TP and DTNRG protocols do not require expensive proprietary hardware at both ends of every connection. DTN already exists in experimental forms [14,17].

In addition, Arctic research networks will need to facilitate mobility and flexibility without user intervention. For example, when an icebreaker approaches the Barrow research facility, it should automatically switch from expensive and low bandwidth INMARSAT and bonded Iridium ${ }^{\mathrm{TM}}$ connections to less expensive, local, higher bandwidth WipLL or WiMAXe connections. DTN is ideal for these applications and for constructing more sophisticated ad hoc mesh networks, possibly with the aid of GPS [18] and probabilistic routing algorithms [19] over a routing channel to incorporate 
hierarchical location-based routing (HLBR) into operational versions of DTN for the Arctic (GPSDTN). The DTN Bundle Protocol is designed specifically for networks where connectivity is characterized by delay or disruption, it incorporates mechanisms for store and forward operations, modular routing algorithms and will work with a companion reliable link protocol (LTP) that can autonomously manage link connections using scheduled or calculated link cues. It is well suited for networks that need to connect mobile wireless devices, including personal communicators, sensor networks, and remote Earth outposts [20].

DTN supports intermittent connectivity, opportunistic or scheduled contacts, long, or variable delays, asymmetric data rates, and high bit error rates, store-and-forward message switching, and Class-of-Service, all of which are necessary in remote areas that are connected by highly dynamic networks. DTN is like current email systems in the sense that each bundle or message has an address, is forwarded toward the recipient via a series of servers (hosts, nodes), each of which stores and keeps trying to forward the message until it either succeeds with its hand-off to the next host or fails to connect and aborts after a set number of attempts [17,21]. DTN does this by placing a bundle layer on top of a "convergence layer" (CL). A CL may be customized for some other network such as TCP/IP or an Iridium ${ }^{\mathrm{TM}}$ raw data channel $[20,22]$. The bundle layer handles bundles also known as messages of arbitrary length. Ideally the length of the bundles is tuned dynamically based on traffic patterns and connectivity dynamics. The bundle header tells the bundle layer how to reassemble the messages after an unknown delay. The DTN Research Group (DTNRG) will incorporate GPSDTN functionality in either the bundle or application layer.

\section{ADAPTING DTN TCP/IP-COMPATIBLE PROTOCOLS TO ARCTIC ENVIRONMENTS}

The topology of an Internet is a list of all pairs of nodes that are known (or at least supposed) to be currently adjacent in the network. While the topology remains unaltered, the route we compute from one node to another will always be the same [setting aside, for the moment, optimizations based on reported fluctuations in data rates, etc.]. Therefore in order to change the route we have to change the topology. In contrast, the topology of a partly mobile ad hoc DTN ("GPSDTN") is a list of all pairs of nodes that we expect to be adjacent (within mutual local broadband radio/optical/acoustic range) over a series of distinct future time intervals; the topology is complemented by a time series of connectivity initiation and termination events. For sparse GPSDTNs in which nodes are usually outside of mutual broadband range some nodes must physically move to transmit data. This means that the route we compute from one node to another must vary over time for data to be exchanged over significant distances.

In the design of the Licklider Transmission Protocol for sparse DTNs [23] termination events are referred to as "link cues". For the operation of spacecraft in orbit one can typically generate all relevant link cues from a schedule based on orbital mechanics to predict where nodes will be at any time. Many Arctic DTNs will be so sparse that they will depend upon the physical movement of some of their nodes to transmit data. In contrast to most interplanetary DTN implementations, however, Arctic nodes will move irregularly in both space and time. Therefore Arctic DTN nodes will need to exchange constantly changing distance and time (velocity) information (GPSDTN link cues) based on GPS (and other GNSS sources) in order to efficiently transfer data from origin to destination.

GPSDTN link cues will be calculated by a hierarchy of regional link cue generators (hierarchical location based "routers" or HLBRs) that will be informed of pending transmission requests (origin, destination, data volume, node status, etc..) by GPSDTN nodes and inform the transmitting node when to begin to attempt data exchange based on the coordinators knowledge of a converging GPSDTN node that have interoperable radios, sufficient buffer and power that are likely to be on or near to the optimum path to the destination. This "routing" information will usually be exchanged via narrowband, long-distance routing channels (Iridium ${ }^{\mathrm{TM}}$ in the Arctic) and via a velocity bundle extension block via restricted local flooding if the narrowband coordinating channel should fail. Subsequent sections describe prototype GPSDTN routing in greater detail.

GPSDTN will develop the new, open-source, DTN protocols necessary to make GPSDTN operational. Several variants of the DTN protocols will be run on each architecture at saturation. The efficiency of transmission will need to be measured for each architecture, location-based algorithm and version of DTN to determine which protocols optimize performance for each architecture and traffic load for each application. Progress has already been made in this direction by the DTNRG. Current experimental implementations of DTN have been written for LINUX-like platforms, Windows and a Symbian-based cell phone in highly customized environments with extremely knowledgeable users.

GPSDTN will use a context-aware (CA) service-oriented architecture (SOA) [24] that can be used to maximize the efficiency of DTNs. The continual evaluation of application and environmental information in a real-time hop-by-hop manner will integrate flexibility into the protocol stack, necessary to adapt to the environmental dynamics. Communicating with a mobile node will benefit from velocityenabled context-awareness, by identifying the declining signal strength as an indicator of loss of line-of-sight and switching communication to another compatible node or alerting network operators to take action.

Practically speaking, simple tools that easily modify common computer operating systems such as Windows $\mathrm{XP}^{\mathrm{TM}}$ and MacOSX ${ }^{\mathrm{TM}}$ to DTN and GPSDTN must be created before DTN can be used widely. These simple tools will be two of many important outcomes from the GPSDTN testbed. We will deploy the sensor and communications network, act as the interface with indigenous communities to gather traditional knowledge regarding climate change, conduct user satisfaction studies and make suggestions to improve GPSDTN. Phase one results of the test bed will then be used to present a wellfounded plan for Phase 2 integration of a circum-arctic GPSDTN for climate change research and sustainability efforts. GPSDTN will not only facilitate much needed Arctic research but is also designed to be an ideal test-bed for DTN 
development. Technologies developed for GPSDTN will have global as well as interplanetary applications $[8,9,20,25]$. For example, an Iridium ${ }^{\mathrm{TM}}$-routing channel could be replaced by a GSM routing-channel for GPSDTNs that are implemented near GSM networks.

Many remote Arctic GPSDTN nodes will be power-limited, especially during the dark winter months. Therefore GPSDTN nodes will be divided into power-rich Active Nodes (A) that are "always-on" to exchange HLBR data via the narrowband routing channels and Passive Nodes $(\mathrm{P})$ that are usually in a sleep mode and use a wake-up beacon from a roving data harvester to activate their Iridium ${ }^{\mathrm{TM}}$ transmitters to conserve power. Active Fixed (AF) power-rich GPSDTN nodes will constantly listen for HLBR data indicating the approach of a mobile node and initiate data exchange. Active Mobile (AM) GPSDTN nodes such as icebreakers, aircraft and snow machines will also constantly update the HLBR routers and AF nodes with their position data and act as data harvesters and freighters. Passive power-limited GPSDTN nodes will usually operate in a low-power listen mode for a wake-up beacon (narrowband, RF, optical or acoustic) before transmitting HLBR data to the routers to update their position/status information. Passive Fixed (PF) power-limited GPSDTN nodes will include remote meteorological stations and other environmental sensors. Passive Mobile (PM) power-limited GPSDTN nodes will include animal biometric and tracking collars, buoys, and researchers on foot among others. AM nodes will activate sleeping PF and PM nodes with wakeup beacons to harvest climate research data and to deliver routine mail and cached web site content for Arctic inhabitants.

\section{NORTHERN SWEDEN AND ICEBREAKER DTN PROTOTYPES - THE FOUNDATION FOR GPSDTN IN THE ARCTIC}

From a technology perspective GPSDTN is an informal subproject of the DTN Research Group (DTNRG). From an Arctic perspective, given the similarity of communications problems throughout the Arctic, the Sami Network Connectivity (SNC) project [3] has adopted the GPSDTN project under the auspices of the Circumpolar Infrastructure Task Force of the Arctic Council and the Northern Forum, which is chaired by the Institute of the North. In addition, the project will be offered as a case study of the Arctic Council Information and Communications Technology Assessment of the Sustainable Development Working Group of the Arctic Council. GPSDTN will be used to improve the connectivity, bandwidth and routing efficiency of wireless networks throughout the Arctic.

The SNC DTN Project is establishing Internet communications for the nomadic Sámi population of reindeer herders who live in remote areas of northern Sweden and who relocate in accordance with a yearly cycle dictated by the behavior of reindeer. This population currently does not have reliable wired, wireless or satellite communication capabilities in most of the areas within which they work and live. The SNC architecture builds on the DTN technology currently being developed by the DTNRG. The basic premise is that a hybrid solution with current Internet technologies should be able to provide basic access to remote and nomadic communities. The initial goal is to provide email, cached web access, reindeer herd tracking telemetry and basic file and data transfer services. The basic design involves using the DTN bundle protocol to relay data between gateways using opportunistic routing through fixed and mobile relays. The mobile relay bundle caches periodically travel between the residential communities, meeting at points where data bundles can be exchanged and at locations where gateways to the Internet are available.

International Research Icebreaker ("Icebreaker") DTN Project seeks to provide connectivity to researchers in the high Arctic on U.S. National Science Foundation icebreakers via bonded Iridium ${ }^{\mathrm{TM}}$ systems and small experimental picosatellites [26]. Such systems are still quite limited in terms of bandwidth and the icebreakers collect terabytes of science data during each research cruise. Standard DTN is sufficient for opportunistic communication between two-such power-rich AM and AF nodes. However, GPSDTN will be used with Iridium $^{\mathrm{TM}}$ to coordinate data exchange between NSF icebreakers, and the BGCCRF and other shore facilities via broadband mobile WiMax connection [2] in the early development phase of GPSDTN in preparation for subsequent use of the icebreakers and BGCCRF with PF nodes such as weather stations and PM nodes such as buoys, animal collars, small UAVs etc.

\section{THE NEED FOR VELOCITY-ENABLED ROUTING IN THE ARCTIC}

Arctic inhabitants need efficient DTNs that conserve connect time, power, buffer space and spectrum. Researchers need to know where their data were generated, when their data were generated, when their data were transmitted, where and when sensor node MACaddressUTM57WVVCCTT2129112288 UTM34WUUZZTT72349678 also known as ("UCThawLake Buoy076") died so that it can be recovered and repaired.

DTN routing efficiency would improve if DTN nodes knew where each other are and are probably going to be (i.e. MoVe and MoVe-Lookahead algorithms [27]. More efficient velocityenabled predictive DTN routing would conserve extremely limited power and expensive and limited connectivity, especially in winter [28]. For example, predictive velocityenabled routing (latitude, longitude, elevation, time-ofposition-fix, broadband communication TX radius, broadband communication type, channel, link quality and residual power and residual buffer) would let DTN icebreakers know when they are within range of power-limited buoys or submarine sensors. Similarly, the new BGCCRF has a single T-1 external GEO satellite connection between Barrow and the rest of the Internet (the thin waist problem) [22]. We need terabyte scale DTN data freighters (GPSDTN nodes on passenger and cargo aircraft) between Barrow and Fairbanks for at least the next several years.

We envision a circum-arctic DTN for research and sustainability efforts that is aided by GPS in which every node has location and universal time information and uses location- 
based forwarding to send packets [28]. Networking reasons for GPSDTN include improved scalability and efficiency of location-based routing due to reduced unidirectional packet flooding [28]. GPSDTN would involve greater routing overhead in the form of location, universal time and infrastructure status information. For many applications such as search and rescue, location-based services and climate change research, however, such "overhead" is as important as the payload ("5 degrees C" or "my snow machine has broken down"). This overhead can be limited to essential updates based on node velocity with data captured from the GPS.

Hierarchical Location-Based Routing Protocols partition the network into groups with the aid of GPS so that every group has a fixed region so that overlap of HLBR routers and HLBR traffic is reduced [28]. We will limit the number of updates to infrequent time-based updates based on node velocity. Supernodes with HLBR tables, Iridium ${ }^{\mathrm{TM}}$ routing channels and external Internet (fiber, copper and occasionally GEO satellite) connectivity will coordinate broadband traffic and use location-based forwarding to send packets directionally between different groups to reduce overhead still further [28]. We will reduce the size of regional velocity-enabled routing tables by adopting hierarchical location-based addressing based on DTN tuples consisting of ordered pairs of Routing Region and Region-Specific components [1].

Our prototype HLBR scheme will define GPSDTN regions by Universal Transverse Mercator grid zone (UTM zones). For example, Lulea, Sweden is in UTM zone 34W. Each UTM zone will have a GPSDTNHLBR router. GPSDTN UTM routing zones will be subdivided into subzones. Each subzone (100,000-Meter Square) has a two-letter ID code ("34WET"). For GPSDTN, each 100,000-Meter Square subzone will be further subdivided on the basis of 10,000-Meter and even 1,000 Meter Squares for GPSDTNs in high population areas with the same naming scheme as necessary. We favor an extended UTM grid reference system because it is a terse and readymade Cartesian coordinate and naming system amenable to the MoVe algortithm as well as MAC-HLBR addressing.

The UTM coordinate system locates any Map Point on earth with a 2 to 10 character geocode. A two digit code implies a precision of $10 \mathrm{~km}$ and a ten digit code corresponds to a $1 \mathrm{~m}$ precision with intermediate steps of $1 \mathrm{~km}, 100 \mathrm{~m}$, and $10 \mathrm{~m}$. It is always displayed in an even number of characters preceded by an alpha-numeric code describing the larger Earth area to which it belongs. For instance, 38 S MC12345678 would be read as: Section: 38, Sub Section: S, Map Section: MC Map Point: 12345678. The map point is broken down further into to equally sized parts of 1, 2, 3, 4 or 5 digits. As with other mapping standards, one reads the map point from West to East first, then from South to North. So 1234 would be the longitudinal equivalent and 5678 would be the latitudinal equivalent. GPSDTN will use the World Geodetic System 1984 earth centered datum because it is the standard datum for GPS.

Therefore a GPSDTN name would consist of the tuple:

Routing Region Component - MAC address plus "home" router consisting of Universal Transverse Mercator (UTM) or Universal Polar Stereographic (UPS) grid zone, Sub Section (100km Square), Map Section (10km square), Local Section (1km square), and Map Point ("MACaddressUTM57WVV CCTT2129112288”).

Region-Specific Component - Current Universal Transverse Mercator (UTM) or Universal Polar Sterographic (UPS) grid zone (DTN Region-Specific), current Subzone, Map Section and Map Point ("UTM34WETZZBB12345678”). UTM zones will be used between 80 degrees South latitude and 84 degrees North latitude. UPS zones will be used south of 80 degrees South latitude and north of 84 degrees North latitude. Current local routers will update each node's authoritative home router for the sake of redundant "fail-over-routing" toward the node's last known location.

Potential vulnerabilities of LBR include routing misbehavior, denial of service attacks, black holes, routing loops, etc. Therefore we need to secure the narrow- and broadband routing channel GPSDTN HLBR information from intentional corruption $[29,30]$. One way to do this would be to encrypt the Iridium $^{\mathrm{TM}}$ or other narrowband links between GPSDTN nodes and their regional HLB routers as well as the intermittent broadband (WiFi, WiMaxE, optical etc..) DTN connections between nodes. A trust model could be established in which each node might receive HLBR information calculated specifically for that node based on a tuple of past information fed to the master control node. This information would be encrypted exclusively by coordinating nodes and meant for a specific subordinate node. This implies a public-private key exchange a priori or some other method where only the two end points know how to decrypt messages. This ensures that LBR information is authentic even if it is received via a transit node. This also means there has to be a registration process that occurs before nodes are deployed and that a lost node could have problems re-connecting to the DTN after a long period of not being available. DTNRG is also enhancing security at the bundle level [31].

\section{Potential Location-BASEd Routing Protocols}

Several potential location-based routing protocols for GPSDTN are available [32]. These include: Location-Aided Routing [33], Distance Routing Effect Algorithm for Mobility [34], Greedy Perimeter Stateless Routing [35], Grid Location Service [36], Priority-based Stateless Geo-routing in Wireless Sensor Networks [37], Terminode Remote Routing [38], Probabilistic Geographic Routing [39], and Motion Vector (MoVe) [26]. We will begin with the MoVe routing protocol and add a three-dimensional capability for aircraft and submersibles, and the ability to compare radio transmission radii and radio types to allow routers to instruct nodes when they are probably within range of other compatible nodes with adequate power and buffer space to begin data exchange.

GPSDTN nodes for Arctic research need to be affordable and therefore must leverage standards and be mass-produced. The National Marine Electronics Association (NMEA) has established widely used navigation data standards that are used by GPS and other types of navigational equipment. For the sake of interoperability we plan to leverage the NMEA 0183 
standard and extend it with a GPSDTN specific NMEA-like sentence that contains narrowband radio type and channel, broadband radio type, radio range, channel number, link quality and residual power in days and residual buffer space in bytes.

These distilled GPSDTN routing sentences will be transmitted over the narrowband velocity routing channel (Iridium $^{\mathrm{TM}}$ in the Arctic) and, optionally, via the intermittent small radius broadband links ( $\mathrm{WiFi}$ and WiMaxE) to the node's local and home HLBR routers (data exchange coordinators)) for the sake of redundancy. The regional HLBRs will use a simple decision tree to identify the next three nodes (predicted waypoints) with sufficient power, buffer and interoperable broadband radios that the originating node will probably encounter and then inform the originating node of their predicted locations. The originating node will calculate its own rendezvous times unless updated by the local HLBR supernode via the narrowband channel. A rendezvous (broadband radio radius) circle will be used by each node to determine when to start and stop attempting to transmit data in order to conserve connect-time, power, spectrum, and buffer. Nodes attempting to transmit data will be updated with regard to the expected locations of the nearest three nodes every five minutes in default mode. We expect many near-misses in sparse Arctic GPSDTNs, hence the requirement to transmit to a default of three potential intermediate nodes unless a return receipt is received from the final destination node beforehand.

Our version zero prototype (Fig. 1) will ingest location, time and waypoint information from the NMEA 0183 v3.1 GGA (Global Position fix data), ZDA (UTC Date/Time and offset), RTE (Waypoints in Active Route), WPL (Waypoint Location), AAM (Waypoint Arrival Alarm), and RMB (Waypoint Velocity) sentences. We will add NMEA-like narrowband and broadband radio, power and buffer fields and distill all of the above to a more compact GPSDTNHLBR routing sentence in anticipation of multiple generations of GPSDTN nodes that use different narrowband (Iridium ${ }^{\mathrm{TM}}$, etc.) and broadband radios (Bluetooth, 802.11a,b,g,n,s, 802.16e etc.). Example narrowband radio codes include: 1 - Iridium $^{\mathrm{TM}}$ Short Burst Data (SBD), 2 - Iridium ${ }^{\mathrm{TM}}$ Short Message Service - Mobile Originated, 3 - Iridium $^{\mathrm{TM}}$ Short Message Service Mobile Terminated, 4 - Global System for Mobile Communications (GSM), 5 - etc... Example broadband radio codes include: 1 - 802.11a, 2 - 802.11b, 3 - 802.11g, 4 $802.11 \mathrm{n}, 5-802.11 \mathrm{~s}, 6-802.16 \mathrm{~d}, 7-802.16 \mathrm{e}, 8$ - Bluetooth 1.0, 9- Bluetooth 1.0B, 10- Bluetooth 1.1, 11- Bluetooth 1.2, 12 - Bluetooth 2.0, 13 - etc... These narrowband and broadband radio codes will be extended and formalized as part of the GPSDTN project.

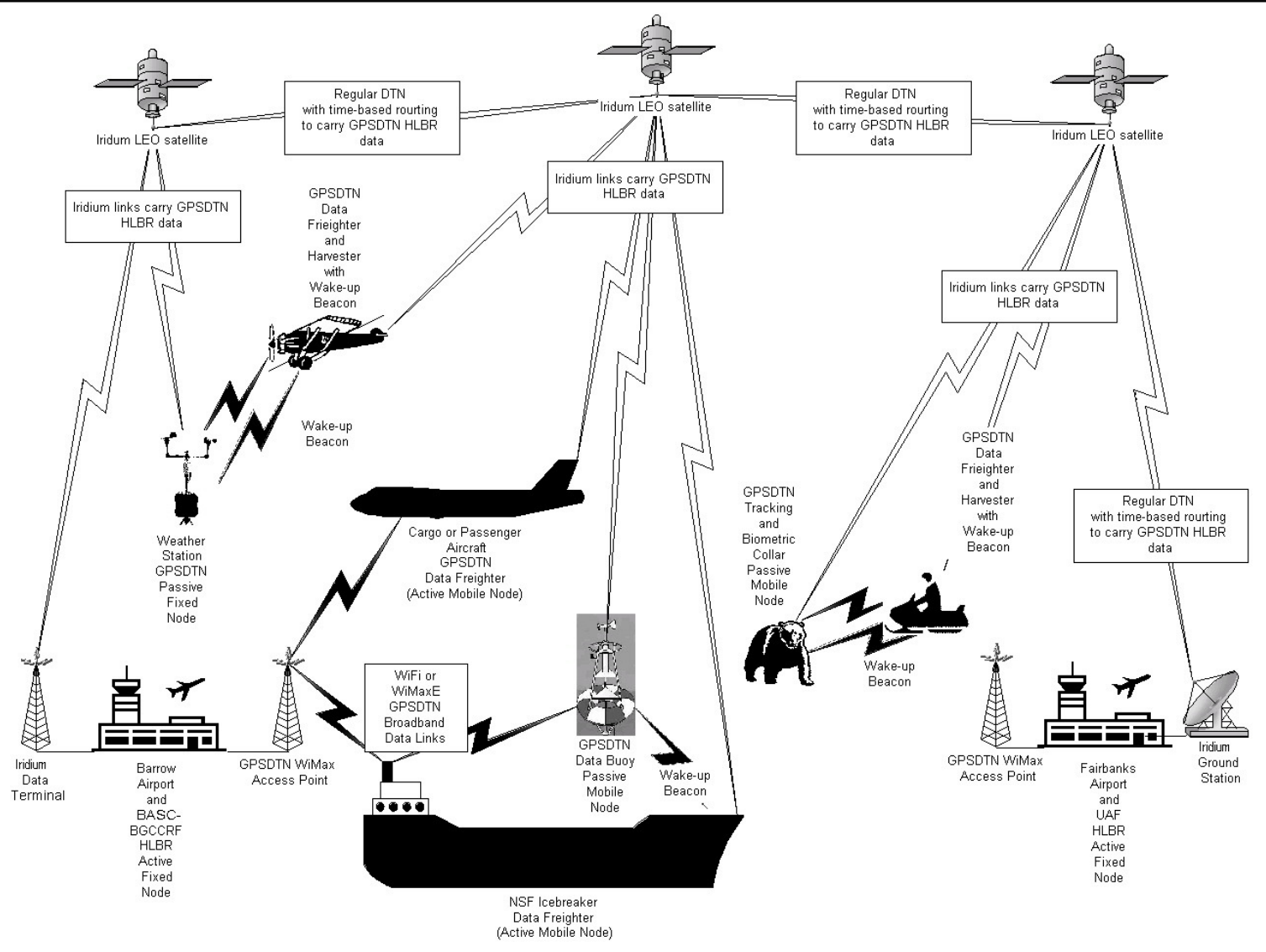

Figure 1. Schematic diagram of GPSDTN on North Slope of Alaska. We envision similar scenarios throughout the Arctic given similar needs and applications of the Northern Forum of Arctic Nations. 
The enhanced three-dimensional MoVe routing algorithm [27] will be used with the GPSDTNHLBR routing sentence distilled from the NMEA navigation sentences and the new "RADIO" sentence fields. The RADIO sentence fields (eventually self-configured by each GPSDTN node) in the GPSDTNHLBR routing sentence will include codes for narrowband and broadband radio type, narrowband and broadband channel, average radio radius in meters, residual power in days, residual buffer space in bytes, link quality and checksum field. The MoVe routing algorithm will be further extended with an energy conservation algorithm [40].

We have defined 58 standard navigation and status elements (fields) for the GPSDTNHLBR routing sentence in anticipation of the rapid growth of GPSDTN. We have intentionally erred on the side of precision, robustness, completeness, interoperability, scalability and extensibility. GPSDTNHLBR will be an OAA compatible variable length string that has a short descriptive flag for each element and commas separating elements to make parsing the string easier.

\section{Fields Extracted from NMEA 0183 sentences}

1) GNSS - NMEA code for Location Device (GP for GPS etc...)

2) LAT - Latitude (deg,decmin,N/S)

3) LON - Longitude (deg,decmin,E/W)

4) GPSQ - GPS Quality Indicator,

5) NSAT - Number of satellites in view, 00 - 12

6) HDOP - Horizontal Dilution of Precision (meters)

7) AAM- Antenna Altitude above/below MSL (geoid) (in meters)

8) AAU - Units of antenna altitude, meters

9) SEP - Geoidal separation, the difference between the WGS-

84 earth ellipsoid and mean-sea-level (geoid), +/-(xxx.xx)

10) SEPU - Units of geoidal separation, meters

11) AGE - Age of differential GPS data, time in seconds since

last SC104 type 1 or 9 update, null field if no DGPS

12) DIFID - Differential reference station ID, 0000-1023

13) UTC - hhmmss.ss = UTC

14) $D-x x=$ Day, 01 to 31

15) $M-x x=$ Month, 01 to 12

16) $Y-X X X X=$ Year

17) DATA - Data status $A=O K, V=$ warning

18) CTE - Cross-track error (nautical miles, 9.9 max.), steer

Left to correct (or $\mathrm{R}=$ right)

19) OWPTID - Origin waypoint ID

20) DWPTID - Destination waypoint ID

21) DWPTLT - Destination waypoint latitude (deg,decmin,N/S)

22) DWPTLN - Destination waypoint longitude ( " E/W)

23) RNG - Range to destination, nautical miles

24) TRU - True bearing to destination

25) HVD - Horizontal velocity towards destination, knots

26) ALRM - Arrival alarm $A=$ arrived, $V=$ not arrived

27) ACE - Arrival circle entered

28) PP - Perpendicular passed

29) CIR - Node's radio TX circle radius in nautical miles

30) WPTN - Waypoint name(s) (NodelDs of next 3 predicted node return nodes in order)

31) NTHID - Identity of nth waypoint (next predicted node)

32) NTHLAT - Latitude of nth waypoint on list and N or S

33) NTHLON - Longitude of nth waypoint and E or W

New Fields for GPSDTNHLBR standard sentence

34) NAM - Node altitude, +/- meters

35) NVV - Node Vertical Velocity towards destination, +/- knots
36) NRT - Narrowband Radio Type

37) BRT - Broadband Radio Type

38) NRC - Narrowband Radio channel

39) BLQ - Broadband Link Quality

40) BRR - Broadband Radio Radius in meters

41) RP - Residual Power in days

42) RB - Residual Buffer in bytes

43) HGRS - Home Grid Reference System (UTM/UPS N/S)

44) HS - Home Section (router/UTM zone)

45) HSS - Home Subsection (router $/ 100 \mathrm{~km}^{2}$ )

46) HMS - Home Map Section (router $/ 10 \mathrm{~km}^{2}$ )

47) HMR - Home Router (router $/ 1 \mathrm{~km}^{2}$ )

48) HMP - Home Map Point

49) CGRS - Current Grid Reference System (UTM/UPS N/S)

50) CS - Current Section (router/UTM zone)

51) CCS - Current Subsection (router $/ 100 \mathrm{~km}^{2}$ )

52) CMS - Current Map Section (router $/ 10 \mathrm{~km}^{2}$ )

53) CLR - Current Local Router (router $/ 1 \mathrm{~km}^{2}$ )

54) CMP - Current Map Point

55) MAC - MAC address

56) NNM - Node Name (“LuleaTechUnivReindeer005”)

via "Node Name Service" built into GPSDTN routers

57) MCS - Mandatory checksum

58) Carriage Return

The GPSDTNHLBR velocity-enabled routing sentence will be transmitted to each regional HLBR router in two ways:

1. As a short message between the originating node and the regional HLBR router via a narrowband routing channel (Iridium ${ }^{\mathrm{TM}}$ in the case of the Arctic). The regional HLBR router calculates the optimum path of nodes ("waypoints") between the source node and the end node to conserve bandwidth, power, and buffer in the overall network. It then transmits a new GPSDTNHLBR sentence to the originating node with the waypoint data. All Active nodes receive updated waypoint information if they are within two nodes or two radio TX radii of the optimum path in terms of distance, power and buffer via short burst data or short message service over Iridium $^{\mathrm{TM}}$. Highly dynamic and sparse DTNs will benefit greatly from this first GPSDTN via narrowband routing channel method.

2. As an extension block in the bundle header of each GPSDTN bundle in case the first method fails and the originating or intermediate nodes must resort to time-based restricted local flooding to find a route to the regional router and destination node. In this way, at least return messages will be able to benefit from the efficiency of HLBR and the regional HLBR router will be able to learn the approximate locations of its nodes.

NMEA 0183 velocity data in the form of the GPSDTNHLBR sentence will need to be recorded for each node and stored in the hierarchy of regional local oracles (HLBR routers) [1] for predictive routing and scientific analyses. If velocity data are not available or do not enable predictive routing due to sudden changes of course, GPSDTN nodes will revert to DTN time-based mode [1] with restricted 
local-floods [38] until they exchange position data with GPSDTN oracles.

Narrowband routing channels will be used to exchange navigation data for HLBR in GPSDTN. NMEA 0183 velocity data for predictive routing will be exchanged via narrowband Iridium $^{\mathrm{TM}}$ channels at predetermined intervals to make sure that GPSDTN nodes attempt to begin data exchange over higher bandwidth local connections as soon as they are probably within range and cease to attempt when probably out of range. For example, a GPSDTN node at Barrow airport will begin to load climate research data onto a plane arriving from Fairbanks as soon as it is instructed to do so by the HLBR router at the BGCCRF in Barrow and cease to do so as soon as the jet moves out of range. A GPSDTN node at Fairbanks airport will off load the data onto fiber optic connections and the rest of the Internet in a similar manner. This conserves bandwidth, connection time, spectrum, power and, most importantly, GPSDTN node buffer space. Only nodes that are likely to be on the path to the destination will be populated with data. A return receipt will be transmitted over the Iridium $^{\mathrm{TM}}$ routing channel to reduce retransmissions. If a return receipt is not received, the default retransmission time will be eight days. GPSDTN will be the parcel post of the Arctic Internet.

GPSDTN nodes will update their positions every seven days or when the have moved $2 / 3$ of their broadband radio radius to reduce narrowband routing channel and (broadband flooding) network traffic and power and buffer consumption $[28,40]$ Iridium $^{\mathrm{TM}}$ already updates velocity oracles for the Arctic +/- 10 kilometers without GPS. Arctic HLBR for DTNs needs GPS for more precision (+/- 10 meters) given the limited range of most broadband radios between GPSDTN nodes. Iridium $^{\mathrm{TM}}$ and partners are developing small portable data units for mobile computers and "motes" with data rates of 9 to 128 kbs. For example NAL Research ${ }^{\mathrm{TM}}$ manufactures an extremely low-power Iridium $^{\mathrm{TM}} / \mathrm{NAL}^{\mathrm{TM}}$ 9601-DGS Iridium L-band Transceiver with GPS that transmits location and time information in accordance with the NMEA 0183 navigation data standard for about $\$ 850$. Slight modification of this unit to include a mobile router that distills the NMEA sentences and adds the radio, power and buffer status fields could populate Local Arctic HLBR GPSDTN routing tables via the narrowband routing channels. We expect the per node cost to decrease as the market for GPSDTN equipment grows over the next several years.

Challenges include: 1. A GPS-enabled routing-based DTN routing protocol (GPSDTN), 2. GPSDTN device development for the Arctic (volume, mass, power limitations (i.e. Juang et al., 2002) and qualification issues), 3. GPSDTN interoperability, and operations issues related to the deployment of the new technology. 4. GPSDTN field user authentication, authorization, and accounting (fairness) issues. 5. GPSDTN survivability in the Arctic environment (polar bears, foxes, icing). 6. GPSDTN mobility (handoffs, security, and overhead).

\section{GPSDTN PROTOTYPE HARDWARE}

GPSDTNHLBR nodes could be prototyped with new, lowcost, shock, moisture and dust resistant laptop computers by Durabook $^{\mathrm{TM}}$ (about \$1,400 each). Prototype Arctic GPSDTN nodes could use the NAL A3LA-DGS (about \$850) for the Iridium narrowband HLBR routing channel connection with little modification. Both units are MIL-STD-810F certified (environmentally hardened). We expect to use $\mathrm{WiFi}$ and WiMaxE for the prototype intermittent broadband radios in GPSDTN. The Durabooks have built-in 802.11b and $g$ networking for the very earliest prototype. With external antennae, early prototype hardware should cost less than $\$ 3,000$ per node before Arctic hardening. Several efforts should make the expansion of GPSDTN even more affordable. Intel is developing a dual mode fixed/moble WiMAX chip set. Several vendors are expected to offer CPEs that will be able to scan for WiFi a,b,g,n and s, WiMax D and E, and EV-DOa connections at several different frequencies. Such scanning consumes power and spectrum but might be reduced with velocity-enabled-routing-data-rich (HLBR) narrowband routing channels coupled with wake-up beacon technologies. Vendors such as Trimble have already integrated GPS with a variety of other narrowband radios that could be used as routing channels in mid to low latitudes where population densities and narrowband routing channel options are greater.

\section{Metrics For Velocity-Enabled, Delay-Tolerant, TCP/IP-COMPATIBLE PROTOCOLS}

The second element of the GPSDTN protocol research is the development of metrics for dynamic mixed-latency networks in order to assess the relative performance of the various protocols on each combination of architecture and infrastructure [39]. We will develop a benchmark procedure that can be used to evaluate all transmission over all the proposed GPSDTN networks and the various protocol developments. Current protocols have difficulty over mixed latency networks, and the networks we propose have short, medium, and long latencies, making GPSDTN the ideal test bed to evaluate DTN protocol efficiencies in mobile architectures.

\section{CONCLUSIONS}

For reasons of cost and performance, the Arctic needs a DTN that can deliver high bandwidth data, especially for the delay tolerant case. A wide collection of people are interested, some of whom are working in the Arctic and/or have experience bringing data to Arctic peoples. GPSDTN is a routing research, development and demonstration effort to create DTNs that are useful for the Arctic and other remote places to enable scientific research, education and commerce with low-cost connectivity.

\section{ACKNOWLEDGMENT}

R.A.B. thanks the DTN Research Group for their assistance with Arctic DTNs for research and sustainability efforts, the Barrow Arctic Science Consortium for travel support and the 
National Science Foundation for research support via Wendy Eisner and Kenneth Hinkel.

\section{REFERENCES}

[1] K. Fall, "A Delay-tolerant network architecture for challenged internets", Intel Research, Berkeley, unpublished (but famous), 2003.

[2] R.A. Beck, K. Fall, S. Burleigh, and D. Pleva, "GPSDTN: Velocity-enabled DTN networks for Arctic Research?"; IETF Delay-Tolerant Networking Research Group, San Diego, Nov. 2006,

[3] M. Udén, M., A. Doria, S. Sjursen, \& A.W. Theodorsen, Anywhere? Anytime? The Northern challenge to ICT developers. In Matilainen, E. (ed.) ICT - Tools for providing information, advice and services for rural SMEs? RUBIES Seminar Proceedings 6.-7. April 2006.

[4] R.A. Beck, W. Eisner, K. Hinkel, H. Pesanti, E. Beiswenger, W. Parke, M. Treadwell, S. Smith, L. Krogh, K.G. Hansen, and V. Peskov, Nutarniq, Uniting the Arctic Community with a Wireless Arctic Network for Circumpolar Communications, Polar Geography, vol. 29, no. 1, pp 1-25, 2005.

[5] K.M., Hinkel, B.M. Jones, W.R. Eisner, C.J. Cuomo, R.A. Beck, and R. Frohn, Methods to Assess Natural and Anthropogenic Thaw Lake Drainage on the Western Arctic Coastal Plain of Northern Alaska, Journal of Geophysical Research, 11 pages, 2007, in press.

[6] M. Johnson, K. Freeman, R. Gilstrap and Beck, R.A., "Networking Technologies Enable Advances in Earth Science", Computer Networks, v. 46, pp. 423-435, 2004.

[7] A. Mainwaring, J. Polastre, R. Szewczyk, D. Culler, and J. Anderson, 2002, "Wireless Sensor Networks for Habitat Monitoring", WSNA 2002.

[8] I. Akyldiz, O. Akan, and J. Fang, "TCP-Planet: A Reliable Transport Protocol for InterPlaNetary Internet", Space Internet Workshop 2003 (SIWIII), Cleveland, Ohio, June 4-6, 2003.

[9] R.A. Beck., et al., "A Space-Based End-to-End Prototype Geographic Information Network for Lunar and Planetary Exploration and Emergency Response (2002 and 2003 Field Experiments)", Computer Networks, vol. 47, pp. 765-783, 2005.

[10] C.E. Mayer., B. E. Jaeger, and D. Kim. "Five years of ACTS propagation studies in Alaska," in: N. Golshan, ed., Proceedings of the Twenty-Third NASA Propagation Experimenters Meeting (NAPEX XXIII). Washington, DC: JPL Publication 99-16, June 2-4, 1999.

[11] T.R. Henderson, and R.H. Katz, "Transport Protocols for Internet-Compatible Satellite Networks", IEEE Journal on Selected Areas of Communications, 1999.

[12] J. Postel, "Transmission Control Protocol", IETF RFC 793, Sept. 1981.

[13] J. Postel, ed., "Internet Protocol-DARPA Internet Program Protocol Specification”, IETF RFC 791, Sept. 1981.

[14] S. Farrell, and V. Cahill, "Delay and Disruption Tolerant Networking", Artech House, to be published in Oct. 2006.

[15] P. Juang, H. Oki, Y. Wang, M. Martonosi, L. Peh, and D. Rubenstein, 2002, "Energy-Efficient Computing for Wildlife Tracking: Design Tradeoffs and Early Experiences with ZebraNet," ASPLOS-X, October 2002.

[16] S. Farrell, S. Symington, and H. Weiss, "Delay-Tolerant Networking Security Overview," IETF Internet draft, draft-irtfdtnrg-sec-overview, work in progress, Mar. 2006.

[17] K. Fall, W. Hong, W. and S. Madden, "Custody Transfer for Reliable Delivery in Delay Tolerant Networks", Intel Research, Berkeley, unpublished, 2003.

[18] Q. Li, and D. Rus, "Communication in Disconnected Ad hoc Networks using Message Relay", Journal of Parallel and Distributed Computing, 2003.

[19] A. Lindgren, A. Doria, and O. Schelen, "Probabilistic Routing in Intermittently Connected Networks", MobiHoc, 2003.
[20] F. Warthman, "Delay-tolerant networks (DTNs) - A tutorial", unpublished, 2003.

[21] K. Scott, and S. Burleigh, "Bundle Protocol Specification," IETF Internet draft, draft-irtf-dtnrg-bundle-spec, work in progress, May 2006.

[22] V. Cerf , S. Burleigh, A. Hooke, L. Torgerson, R. Durst, K. Scott, K. Fall, H. Weiss "Delay-Tolerant Network Architecture," IETF Internet draft, draft-irtf-dtnrg-arch, work in progress, Mar. 2006.

[23] S. Farrel, M. Ramadas, S. Burleigh, "Licklider Transmission Protocol - Extensions", Delay Tolerant Networking Research Group, Internet Draft, Work-in-Progress, September, 20, 2006.

[24] Peoples, C; Parr, G; Scotney, B; Moore, A; Dini, P; "Improving the Performance of Asynchronous Communication in LongDistance Delay-Sensitive Networks through the Development of Context-Aware Architectures", Proceedings of the IEEE International Conference on Autonomic and Autonomous Systems, vol. 19-21, pp. 49-57, July 2006.

[25] Z. Zhang, "Routing in intermittently connected mobile ad hoc networks and delay tolerant networks: overview and challenges", IEEE Comm. Surveys and Tutorials, vol. 8, no. 1, pp. 24-37, 2006.

[26] D. Forcucci and D.N. Chayes, "Ocean Observations in the Arctic From the US Coast Guard Icebreaker Healy", AGU, Ocean Sciences, 2006.

[27] J. LeBrun, C-N Chuah, and D. Ghosal, "Knowledge-Based Opportunistic Forwarding in Vehicular Wireless Ad Hoc Networks," IEEE Vehicular Technology Conference, May/June 2005.

[28] Y. Peng, "Scaleable Routing in MANET", Proceedings of CeMNet, Nanyang Technological University, Singapore, 2003.

[29] Z. Zhou, "Securing Location-Based Routing in MANET", Proceedings of CeMNet, Nanyang Technological University, Singapore, 2003.

[30] T. Roosta, S. Shieh, S. Sastry, "Taxonomy of security attacks in sensor networks and countermeasures", IEEE, Mobicom 2005.

[31] S. Symington, S. Farrell, and H. Weiss, "Bundle Security Protocol Specification," IETF Internet draft, draft-irtf-dtnrgbundle-security, work in progress, Mar. 2006.

[32] S. Giordano, I. Stojmenovic, L. Blazevic, "Position-based routing algorithms for ad hoc networks: a taxonomy"; unpublished, 2003.

[33] Y-B. Ko, and N.H. Vaidya, Location-aided routing (LAR) in mobile ad hoc networks, Wireless Networks vol. 6, pp.307-321, 2000.

[34] S. Basagni,, I. Chlamtac., R. R. Syroutiuk, and B.A. Wooward, "Distance routing effect Algorithm for mobility (DREAM)", MobiCom '98, pp. 76-84, October 1998.

[35] B. Karp, and H.T., Kung, "GPSR: Greedy perimeter stateless routing for wireless networks", MobiCom2000, pp. 243-254, August 2000.

[36] J. Li, J., Janotti, S. Douglas, J., De Coutu., D. Karger,and R. Morris, GLS (Grid) (Geographic Location Service) - "Scalable location service for geographic ad hoc routing", unpublished, M.I.T. Laboratory for Computer Science, 2000.

[37] Y. Xu; W.-C. Lee; J. Xu; G. Mitchell, "PSGR: priority-based stateless geo-routing in wireless sensor networks", Proceedings of Mobile Ad hoc and Sensor Systems Conference, Nov. 2005.

[38] L. Blazevic, J-Y. Le Boudec, and S.A. Giordano, "A location based routing method for mobile ad hoc networks". IEEE Transactions on Mobile Computing, vol. 3, no. 4, pp. 97-110, December 2005.

[39] T. Roosta, "Probabilistic geographic routing protocol for ad hoc and sensor networks", Proceeding (474) Wireless Networks and Emerging Technologies, 2005.

[40] S. Jain, K. Fall, and R. Patra, 2004, "Routing in a Delay Tolerant Network" ICSI/ICIR - 12 May, 2003. 